\title{
Ansatz zur ganzheitlichen Erstellung und Verarbeitung von Dienstleistungsmodellen
}

Heiko Kern, Martin Böttcher, Stefan Kühne und Kyrill Meyer

Obgleich die Notwendigkeit einer Dienstleistungsmodellierung ausreichend anerkannt ist, wird ein Fehlen einer ganzheitlichen Modellierungsmethode weiterhin bemängelt. Einen Grund für dieses Fehlen sehen die Autoren darin, dass bei der Erstellung von Dienstleistungsmodellen die Metamodellebenen oftmals nicht klar genug getrennt werden. Eine solche Trennung kann zu einer wesentlichen Verbesserung der Ergebnisse bei Forschungsbemühungen in der Domäne der Dienstleistungsmodellierung führen. Gleichzeitig impliziert eine Betrachtung von Metamodellebenen die Notwendigkeit der Interoperabilität auf der Ebene der Metametamodelle. Dieser Beitrag legt die Gründe für die Notwendigkeit einer klaren Betrachtung der Metamodellierungsebenen dar und zeigt die Möglichkeit eines Mappings auf der Ebene der Metametamodelle als elementares Gestaltungselement einer ganzheitlichen Modellierungsmethode auf.

\section{$1 \quad$ Einleitung}

Das Forschungsgebiet der Dienstleistungsmodellierung weist generell einige Divergenzen auf. So wird einerseits eine ganzheitliche domänenspezifische Modellierungsmethode für den Dienstleistungsbereich gefordert (Alonso-Rasgado et al. 2004, O'Sullivan 2006). Andererseits wird bis heute konstatiert, dass eine ganzheitliche Modellierungsmethodik nicht existiert (Grieble 2004, O'Sullivan 2006). Vielmehr werden in der wissenschaftlichen Literatur verschiedene singuläre Modellierungsansätze für die Dienstleistungsdomäne vorgestellt, welche von praxisnahen Projektergebnissen (Thomas und Scheer 2003) bis hin zu wissenschaftlichen Ergebnissen reichen, beispielsweise in Form publizierter Dissertationen (Hermsen 2000). Keiner dieser Ansätze erfüllt jedoch die Kriterien einer detaillierten Ganzheitlichkeit in vollem Umfang.

Des Weiteren wird in der Dienstleistungsdomäne bereits seit Langem bemängelt, dass der phasenübergreifende Einsatz von Werkzeugen unzureichend gelöst ist. Dies bedeutet beispielsweise, dass der Einsatz von Modellierungswerkzeugen vorwiegend auf die Designphase beschränkt ist (Heckmann et al. 1998) und somit 
eine Wiederverwendung der erstellten Modelle in anderen Phasen nicht möglich ist.

Ausgehend von dieser Situation wird dem Artikel die Hypothese zugrunde gelegt, dass es eines ganzheitlichen Ansatzes zur Modellierung von Dienstleistungen und Verarbeitung von Dienstleistungsmodellen bedarf. Die Ganzheitlichkeit soll sich aber nicht nur auf die beiden Aspekte der Modellierung von Dienstleistungen und Verarbeitung von Dienstleistungsmodellen beziehen, sondern auch auf die Definition von Modellierungssprachen für die Beschreibung von Dienstleistungen. Dabei ist es in diesem Beitrag nicht das Ziel, eine konkrete ganzheitliche Modellierungsmethode zu definieren, sondern lediglich die Voraussetzungen für eine ganzheitliche Modellierung darzulegen.

Um die geforderte Ganzheitlichkeit in der Dienstleistungsdomäne zu realisieren, wird eine strikte Trennung von Metamodellebenen in der Dienstleistungsmodellierung vorgeschlagen. Darauf aufbauend wird ein Ansatz vorgestellt, der auf einem Mapping zwischen Metametamodellen basiert und dadurch den Austausch von Modellen und dazugehörigen Metamodellen zwischen verschiedenen Werkzeugen ermöglicht. Dieser Ansatz wird im weiteren Verlauf als „M3-Levelbasierte Brücke" bezeichnet.

Im Folgenden wird zunächst auf die Notwendigkeit eines ganzheitlichen Ansatzes in der Dienstleistungsmodellierung eingegangen. Anschließend wird im Abschnitt 3 die Anwendung der Metamodellebenen als Ansatz zur Ganzheitlichkeit in der Dienstleistungsdomäne beschrieben. Aufbauend darauf wird im Abschnitt 4 das Konzept des Technikraums beschrieben, um dann im darauf folgenden Abschnitt 5 den Ansatz der M3-Level-basierten Brücke vorzustellen. Abschließend wird die Machbarkeit des Ansatzes am Beispiel der Architektur integrierter Informationssysteme (ARIS) (Scheer 2002) und dem Eclipse Modeling Framework (EMF) (Budinsky et al. 2004) demonstriert.

\section{Notwendigkeit eines ganzheitlichen Ansatzes}

Die Notwendigkeit einer ganzheitlichen domänenspezifischen Modellierungsmethode für den Dienstleistungsbereich ist weiterhin unumstritten (Alonso-Rasgado et al. 2004, O'Sullivan 2006). Innerhalb der letzten Jahre haben sich daher zahlreiche verschiedene Modellierungsansätze zur Beschreibung von Dienstleistungen herausgebildet. Deren Differenzen sind insbesondere mit der Komplexität von Dienstleistungen sowie der breit aufgestellten Dienstleistungsforschung zu erklären. So müssen beispielsweise verschiedene Phasen (Ideenfindungs-, Anforderungs-, Design- und Einführungsphase), Rollen mit unterschiedlichen Fähigkeiten und Kenntnissen sowie weitere Aspekte bei der Modellierung berücksichtigt werden.

Die existierenden Ansätze fokussieren dabei entweder einen Teilaspekt (beispielsweise nicht-funktionale Eigenschaften) und exkludieren somit eine gesamtheitliche Betrachtung oder sie konzentrieren sich auf das gesamte Spektrum der 
Dienstleistungsmodellierung bei gleichzeitiger höherer Abstraktion (beispielsweise Grieble 2004, Hermsen 2000). Diese Unterschiede liegen oft in der individuellen Motivation der Dienstleistungsmodellierung begründet. So wurden Dienstleistungsmodelle beispielsweise als das adäquate Mittel für ein eigentlich modellierungsunabhängiges Ziel betrachtet. Grieble (2004) definiert in seiner Arbeit ein Modell, auf welchem aufbauend ein Benchmarking von Dienstleistungen durchzuführen ist. Hermsen (2000) hingegen verfolgt das Ziel einer kundenindividuellen Konfiguration, welche auf einem entsprechenden Modell aufzubauen ist. Gegenüber solchen Ansätzen existieren Arbeiten, welche explizit die Modellierung von Dienstleistungen unabhängig von dem letztendlichen Einsatzzweck betrachten. Hierzu zählen beispielsweise die schon seit Langem vorhandenen Modellierungsansätze von Shostack $(1982,1984)$, bei welchen Dienstleistungen entsprechend ihrer prozessualen Eigenschaft und entsprechend der Notwendigkeit der Integration von Produkten betrachtet werden. Letztlich existieren auch Modelle, resultierend aus unterschiedlichen Projektergebnissen, wie beispielsweise REBECA (Thomas und Scheer 2003) oder die PAS 1018 (Mörschel und Hoeck 2002).

Obgleich die existierenden Modelle unterschiedlich motiviert sind, lässt sich doch feststellen, dass viele von ihnen entweder direkt auf der Triade von Prozess-, Ressourcen- und Ergebnismodell aufbauen oder sich in diese einpassen lassen. ${ }^{1}$ Diese Dreiteilung, unter anderem proklamiert von Bullinger et al. (2003), baut auf den originär modellierungsunabhängigen Arbeiten von Meffert und Bruhn (2006) auf.

Um eine Dienstleistung in ihrer Gesamtheit beschreiben zu können, bedarf es eines ganzheitlichen Ansatzes, welcher die einzelnen Aspekte einer Dienstleistungsbeschreibung (Triade) mit einem adäquaten Detailliertheitsgrad abdeckt. Selbst wenn die einzelnen Aspekte zu unterschiedlichen Modellierungsmethoden führen, müssen die Modellierungsmethoden doch untereinander verbunden sein, um den zwischen diesen Methoden existierenden Interdependenzen gerecht zu werden. So werden beispielsweise sowohl im Produkt- als auch im Prozessmodell gleiche Begriffe verwendet, die als solche auch erkennbar sein müssen.

Neben der Berücksichtigung von Interdependenzen zwischen verschiedenen Modellen existiert bislang kein einheitlicher respektive zentraler Ansatz zur Dienstleistungsmodellierung, welcher sukzessive weiterentwickelt werden kann. Vielmehr stellen viele der analysierten Arbeiten immerwährende Neuanfänge im Bereich der Dienstleistungsmodellierung dar. Diese Nichtexistenz ist unter anderem der Tatsache unterschiedlicher Formalisierungsgrade einzelner Modellierungsvorschläge geschuldet. Während einige Arbeiten die Ergebnisse rein textuell ausformulieren und kaum erkennbare Formalismen darlegen, nutzen andere Entwürfe jeweils unterschiedliche Formalisierungsansätze, ohne diese immer explizit

1 Die Analyse von 12 Modellierungsansätzen (dargelegt in 25 Dokumenten) sowie von 20 Standardwerken der Dienstleistungsforschung ergab, dass die proklamierte Triade von Prozess, Ressourcen und Ergebnis generell verfolgt werden kann, wenn diese um die Betrachtung der Modularisierung erweitert wird. 
darzulegen. Hierdurch sind einerseits der Vergleich der unterschiedlichen Modellierungsansätze und andererseits die strukturierte Weiterentwicklung erschwert.

Ein weiterer Aspekt bei der Betrachtung eines ganzheitlichen Ansatzes zur Dienstleistungsmodellierung ist die Verbesserung hinsichtlich einer ganzheitlichen Verarbeitung von Modellen durch entsprechende Werkzeuge. Daher ist es entscheidend, Modelle so zu definieren, dass Modelle derart zu definieren sind, dass Modelloperatoren adäquat auf ihnen angewendet werden können.. Hierfür besteht die Notwendigkeit gut strukturierter Modelle mit einer klaren Definition der genutzten Sprachen.

\section{Metamodellebenen in der Dienstleistungsmodellierung}

Als Ansatz für eine ganzheitliche Modellierung wird die strikte Trennung von Metamodellebenen, wie sie in Abb. 1 dargestellt sind, vorgeschlagen. Eine zu betrachtende Dienstleistung, auch als „system under study“ (SUS) bezeichnet, bildet dabei den Ausgangspunkt. Sie wird unter Verwendung einer geeigneten Modellierungssprache durch ein Modell beschrieben. Die in der Modellierungssprache enthaltenen Sprachkonzepte (abstrakte Syntax) werden in einem dazugehörigen Metamodell repräsentiert ( $\mu$-Relation) (Favre 2005a, Mellor et al. 2004, Kühne 2005).

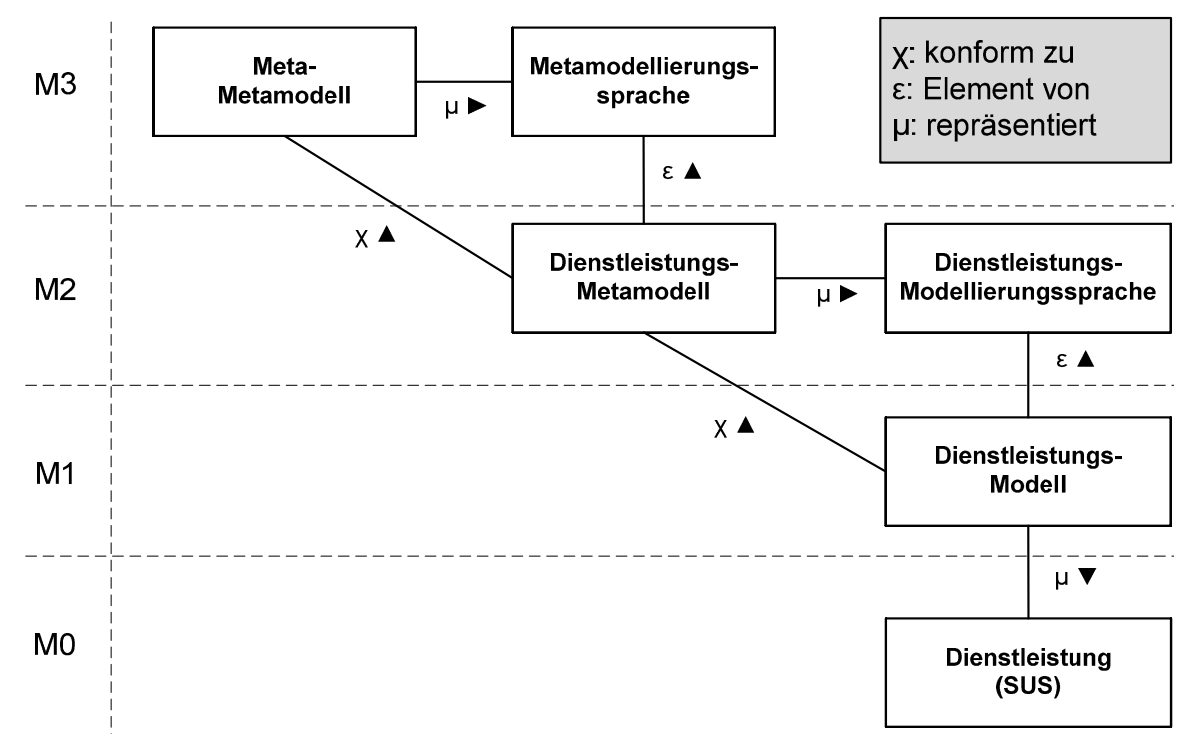

Abb. 1. Metamodellebenen in der Dienstleistungsmodellierung (in Anlehnung an Favre 2005a) 
Metamodelle können wiederum mit einer Sprache beschrieben werden, wobei die enthaltenen Sprachkonzepte (abstrakte Syntax) ebenfalls in einem Metamodell, dem so genannten Metametamodell, repräsentiert werden. Modell, Metamodell und Metametamodell stehen in einer ,konform-zu-Beziehung“ ( $\chi$-Relation) (Favre 2005a, Bézivin 2005, Kühne 2005), die auch als Instanz- oder Ausprägungsbeziehung bezeichnet wird, und bilden eine Metamodellhierarchie. Diese Hierarchie setzt sich aus den vier Ebenen M0-M3 zusammen, wobei M0 die Ebene des Systems und M3 die Metametamodellebene darstellt. Im Allgemeinen besteht eine Hierarchie aus vier Ebenen (Tolvanen 1998).

Die Anwendung beziehungsweise die Beachtung der Metamodellierungsebenen führt aus folgenden Gründen zu einer Verbesserung der Anstrengungen im Bereich der Dienstleistungsmodellierung:

1. Ansätze im Bereich der Dienstleistungsmodellierung sind durch die Berücksichtigung der Metamodellebenen gezwungen, explizit eine der Ebenen (sehr wahrscheinlich die M2-Ebene) zu betrachten. Die aus der Missachtung dieser Ebenen resultierende Heterogenität existierender Ansätze kann damit in $\mathrm{Zu}-$ kunft vermindert werden.

2. Die erstellten Dienstleistungsmetamodelle weisen eine Konformität zu einem gemeinsamen Metametamodell auf und gewährleisten die Weiterentwicklung unter Beibehaltung einer solchen Konformität, so dass eine strukturierte Fortführung von Ansätzen möglich ist. Die Weiterentwicklung von Dienstleistungsmetamodellen wird durch die Offenlegung des explizit genutzten Metametamodells verbessert.

3. In Abhängigkeit des ausgewählten Metametamodells können Relationen zwischen Metamodellen definiert werden, um somit Abhängigkeiten beziehungsweise Beziehungen zwischen Modellen darzustellen.

4. Die ganzheitliche Verarbeitung von Modellen wird verbessert, da entsprechende Werkzeuge implementiert werden können, die auf der Struktur des Metametamodells aufbauen. Somit können beispielsweise spezifische Modelloperationen, wie Transformation, Validierung oder Analysen, in Abhängigkeit der Metamodelle definiert werden.

\section{Technikräume für die Dienstleistungsmodellierung}

Das vorgestellte Konzept der Metamodellhierarchie, welche aus drei Modellierungsmetaebenen besteht, ist nicht neu und wird in verschiedenen Technologien umgesetzt. Eine solche Umsetzung kann als Technikraum (Kurtev et al. 2002, Favre 2005b, Bézivin et al. 2005b) bezeichnet werden, wobei der Begriff vorwiegend seine Verwendung im Bereich des Model-driven Engineering (MDE) (Bézivin 2005, Favre 2005b) findet. Ein Technikraum wird oftmals mit bestimmten Ideen, Konzepten, Werkzeugen, Möglichkeiten und erforderlichen Fähigkeiten 
verbunden, um Lösungsansätze für spezifische Problemstellungen zu bieten. Weiterhin kann ein Technikraum oft mit einer Gruppe beziehungsweise Gemeinschaft assoziiert werden, in der ein gewisser Konsens hinsichtlich des Wissensgebietes beziehungsweise der akzeptierten Literatur herrscht und welche sich unter anderem über Konferenzen und Workshops austauscht.

Technikräume sind für verschiedene Aufgabenbereiche unterschiedlich gut geeignet. Diese Aufgaben können unter anderem die Modellierung, Transformation, Speicherung, Analyse oder der Austausch von Modellen sein. Die Entscheidung für einen geeigneten Raum kann dabei auf Basis unterschiedlicher Kriterien, wie dem zugrunde liegende Kalkül oder den bereits existierenden Werkzeugen, getroffen werden. So benötigt beispielsweise die Beschreibung der Prozesssicht die Repräsentation von Graphen oder aber im Falle deklarativer Beschreibung die Abbildung von Regeln. Dahingegen benötigt die Aufstellung von Hierarchien Kalküle zur Repräsentation von Bäumen.

Im Folgenden werden verschiedene Technikräume betrachtet, in denen das Konzept der Metamodellhierarchie wieder zu finden ist, und eine mögliche Einordnung in einen der zuvor aufgezählten Aufgabenbereiche vorgeschlagen:

- Architektur integrierter Informationssysteme: Als technischer Raum stellt ARIS eine Reihe von Konzepten und Werkzeugen zum Geschäftsprozessmanagement bereit. Hierzu zählt unter anderem das ARIS-Haus, welches Geschäftsprozesse in verschiedene Sichten und Abstraktionsebenen unterteilt. Als Werkzeugsammlung steht die ARIS-Plattform zur Verfügung. Die Metamodellhierarchie besteht aus dem A3-Modell (Kern und Kühne 2007a), Methodenfiltern und ARIS-Modellen. Aufgrund des Sichten- und Ebenenkonzepts und den dazugehörigen Modellierungswerkzeugen wird ARIS vor allem im Modellierungsbereich angewendet.

- Eclipse Modeling Framework: Das Eclipse Modeling Framework ist ein OpenSource-Framework, das die Entwicklung von Java-Anwendungen unterstützt und als Plug-In für die Eclipse-Plattform zur Verfügung steht (Budinsky et al. 2004). Die Metamodellhierarchie besteht aus Ecore, EMF-Metamodellen und EMF-Modellen. Aufbauend auf dem Framework existiert eine Vielzahl an Werkzeugen für die Modellverarbeitung (Efftinge 2006, Efftinge und Kadura 2006, Jouault und Kurtev 2005, IBM 2004, Biermann et al. 2006), weshalb EMF in der Modellverarbeitung eingesetzt werden kann.

- Extensible Markup Language: Die Extensible Markup Language (XML) (W3C 2006) ist eine vom W3C standardisierte Sprache zur Definition von MarkupSprachen für die Beschreibung von strukturierten und semistrukturierten Daten in Form von XML-Dokumenten. Aufbauend auf dem XML-Standard existiert eine Vielzahl von weiteren Standards für die Verarbeitung von XML-Dokumenten, wie XSLT, XPATH oder XQuery. Die Metamodellhierarchie im XMLRaum besteht aus der XML-Spezifikation, XML-Schemas/DTDs und XML- 
Dokumenten. XML hat sich als Format für die Modellverarbeitung und den Austausch von Modelldaten durchgesetzt.

- Web Ontology Language: Die Web Ontology Language (OWL) (W3C 2004) ist ebenfalls eine Spezifikation des W3C, um Ontologien zu beschreiben, zu publizieren und zu verteilen. OWL ist ein wesentlicher Bestandteil der SemanticWeb-Initiative und eignet sich zur formalen Definition von Konzepten und deren Beziehungen. Aufbauend auf der formalen Beschreibung existieren Werkzeuge, wie Reasoner, welche entsprechende Interferenzen bestehender Konzepte berechnen und neues Wissen ableiten können. Die Metamodellhierarchie im OWL-Raum besteht aus der OWL-Spezifikation, OWL-Konzepten und OWLInstanzen.

\section{Ganzheitlichkeit durch M3-Level-basierte Brücken}

Die Existenz verschiedener Technikräume und deren unterschiedliche Eignung bezüglich bestimmter Aufgabenbereiche führen im Rahmen der Dienstleistungsmodellierung dazu, dass Metamodelle und Modelle in unterschiedlichen Räumen entwickelt werden. Beispielsweise ist es vorstellbar, dass sich der ARIS-Raum für eine bestimmte Dienstleistungsdomäne aufgrund seiner existierenden Modellierungssprachen (beispielsweise EPK), bereits vorhandenen Werkzeugen und dem zugrunde liegenden Kalkül (die Repräsentation von Graphen) zur Beschreibung von Prozessmodellen eignet. Hingegen bietet sich unter Umständen der OWLRaum für die Entwicklung von Produktmodellen an, da in OWL entsprechende Klassenstrukturen, wie sie in Produktmodellen vorkommen, abgebildet und mit speziellen Werkzeugen erstellt und überprüft werden können.

Die Eignung beziehungsweise Verwendung verschiedener Technikräume für die Dienstleistungsmodellierung erschwert die geforderte ganzheitliche Betrachtung. Um diese dennoch zu erreichen, wird eine Überführung der Modelle und Metamodelle zwischen den verschiedenen Räumen durch den Ansatz der M3-Levelbasierten Brücken vorgeschlagen. Dadurch wird beispielsweise die Weiterentwicklung auf dem Gebiet der Dienstleistungsmodellierung sichergestellt, da Metamodelle in unterschiedlichen Räumen miteinander vergleichbar gemacht werden können. Des Weiteren kann eine Überführung von Modellen in andere Räume hilfreich sein, um dessen Funktionalität bei der Modellverarbeitung zu nutzen.

M3-Level-basierte Brücken wurden bereits für verschiedene Metamodellhierarchien entwickelt (Bézivin et al. 2005a, Bézivin et al. 2005b, Kern und Kühne 2007a, Duddy et al. 2003, Wimmer et al. 2006). Auch wenn die Implementierungen solcher Brücken durchaus unterschiedlich sein können, beruhen sie auf der derselben konzeptionellen Grundlage. Ausgangspunkt ist zunächst die Berücksichtigung der Metamodellebenen, wie sie in Abb. 2 dargestellt sind. Aufbauend darauf müssen semantisch ähnliche Konstrukte auf M3-Ebene identifiziert werden und eine Abbildung zwischen diesen Metasprachkonzepten gefunden werden. Auf Basis der Abbildung auf M3-Ebene können konkrete Sprachkonzepte durch eine 
Transformation auf M2-Ebene abgebildet werden. Somit ist eine Abbildung der Metamodelle der beiden Räume sichergestellt. Eine solche Transformation kann unidirektional oder bidirektional sein. Erstere bedeutet, dass nicht alle Metasprachkonzepte des einen Metametamodells auf die des anderen abgebildet werden können. Somit ist es nur möglich, Sprachdefinitionen von einem Modellierungsraum in den anderen zu überführen, jedoch nicht umgekehrt beziehungsweise nicht vollständig umgekehrt. Eine bidirektionale Abbildung hingegen erfordert die Abbildung aller Metasprachkonzepte aufeinander und ermöglicht die Überführung von Sprachdefinitionen in beide Richtungen. Nach der Definition der Abbildung auf M3-Ebene und der Ableitung der Transformation auf M2-Ebene müssen nun Transformationsregeln auf M1-Ebene definiert werden. Dadurch können Modelle zwischen den Räumen transformiert werden. Ausgangspunkt sind wieder die Abbildungsregeln auf M3-Ebene. Weiterhin muss wie bei der M2-Ebenen-Transformation bekannt sein, auf welche Weise Metasprachkonzepte auf M1-Ebene ausgeprägt werden.

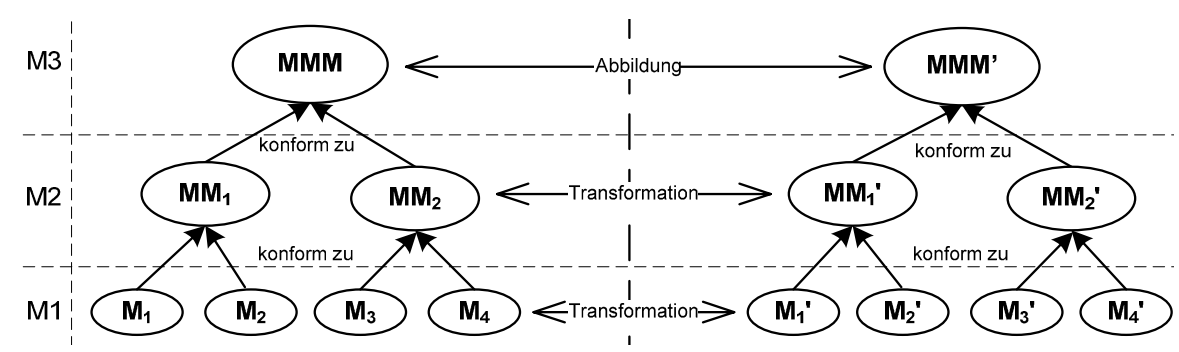

Abb. 2. M3-Level-basierte Brücke

\section{Machbarkeitsanalyse am Beispiel von ARIS und Eclipse EMF}

Der vorgeschlagene Ansatz zur ganzheitlichen Erstellung und Verarbeitung von Dienstleistungsmodellen soll in diesem Abschnitt anhand von ARIS und Eclipse EMF gezeigt werden. Wie bereits im Abschnitt 4 beschrieben, kann ARIS als ein Technikraum aufgefasst werden, der vorwiegend im Geschäftsprozessmanagement seine Anwendung findet. Ausgewählte ARIS-Modellierungssprachen können aber auch im Bereich der Dienstleistungsmodellierung angewendet werden (Scheer et al. 2002). Durch die Möglichkeit der Hinterlegung von Modellobjekten können Beziehungen zwischen den verschiedenen Modellen dargestellt werden, um dadurch eine ganzheitliche Betrachtung zu gewährleisten. Diese ganzheitliche Modellierung wird durch die Einhaltung des im ARIS vorhandenen A3-Modells (Metametamodell), welches in Abb. 3 dargestellt ist, und der Metamodellebenen gewährleistet. Weiterhin sind alle Modelle in einem vom A3-Modell abgeleiteten 
Modell-Repository (Kern und Kühne 2007a) abgelegt, wodurch eine ganzheitliche Modellverarbeitung durch ARIS-Skript möglich ist.

Ein Technikraum, der sich aufgrund offener Standards, frei verfügbarer OpenSource-Implementierungen und zahlreicher Werkzeuge für die Verarbeitung von Modellen empfiehlt, ist durch Eclipse EMF gegeben. Beispielsweise existieren für die Umsetzung von Modell-zu-Modell- und Modell-zu-Text-Transformationen zahlreiche Werkzeuge. Diese basieren auf unterschiedlichen Transformationsansätzen, wie dem graphbasierten Ansatz AGG (Biermann et al. 2006), dem relationalen Ansatz ATL (Jouault und Kurtev 2005) oder MTF (IBM 2004), dem funktionalen Ansatz XTend (Efftinge 2006) oder dem template-basierten Ansatz XPand (Efftinge und Kadura 2006). Im Vergleich zum imperativen Ansatz, auf dem beispielsweise ARIS-Skript basiert, ergeben sich durch diese Ansätze Verbesserungspotenziale hinsichtlich Modularität, Anpassbarkeit, Wiederverwendbarkeit oder Benutzbarkeit. Dies war die Motivation für eine M3-Level-basierte Brücke zwischen ARIS und Eclipse EMF, die es ermöglicht, Modelle und Metamodelle von ARIS nach Eclipse EMF zu überführen, die überführten Modelle mit EMF-Werkzeugen zu bearbeiten und anschließend die Modelle wieder in den ARIS-Raum zu überführen.

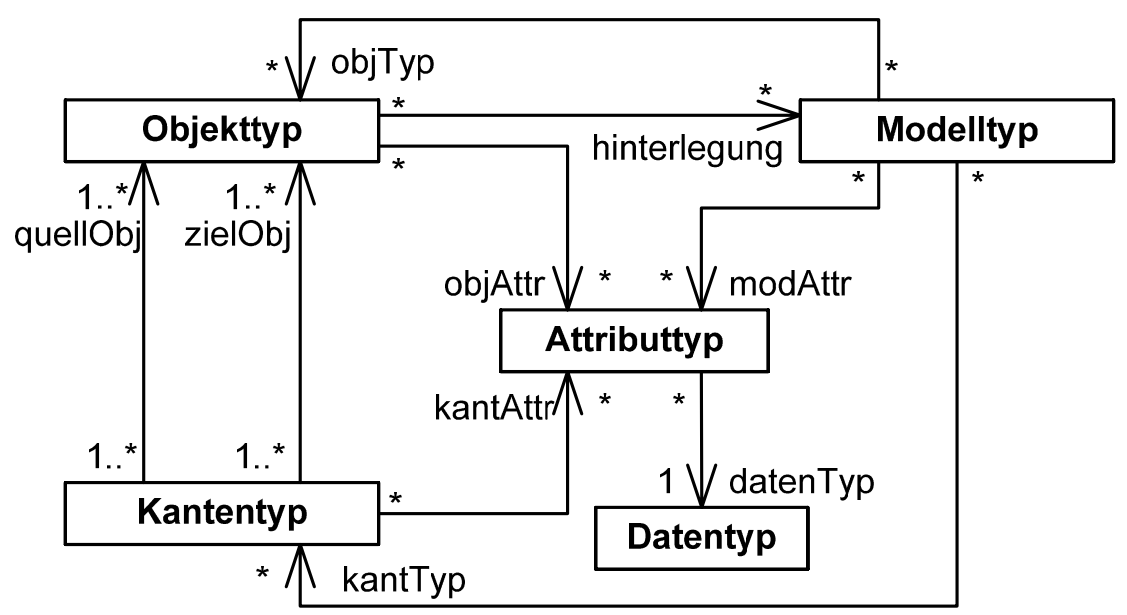

Abb. 3. Metametamodell von ARIS: A3-Modell

Die Brücke bildet zunächst semantisch ähnliche Elemente zwischen dem A3Modell von ARIS und dem Ecore-Modell von Eclipse EMF aufeinander ab (vgl. Abb. 4). Eine Regel ist beispielsweise die Abbildung von Objekttyp auf EClass, wobei der Name gleich ist. Eine weitere Regel ist die Abbildung von Attributtyp auf EAtttribute. Eine detaillierte Beschreibung der entsprechenden Abbildungsregeln wird von Kern und Kühne (2007a) dargelegt. Aufbauend auf den Abbildungsregeln können konkrete Transformationsregeln auf M2-Ebene und M1-Ebene definiert werden. Dadurch können Metamodelle und Modelle zwischen den beiden 
Metamodellhierarchien transformiert werden. Somit besteht beispielsweise die Möglichkeit, wie bereits in Kern und Kühne (2007b) beschrieben, ein Prozessmodell, welches in ARIS-EPK-Notation beschrieben wurde, nach Eclipse EMF zu überführen, um dieses Prozessmodell anschließend mit der ATLAS Transformation Language (ATL) (Jouault und Kurtev 2005) zu validieren.

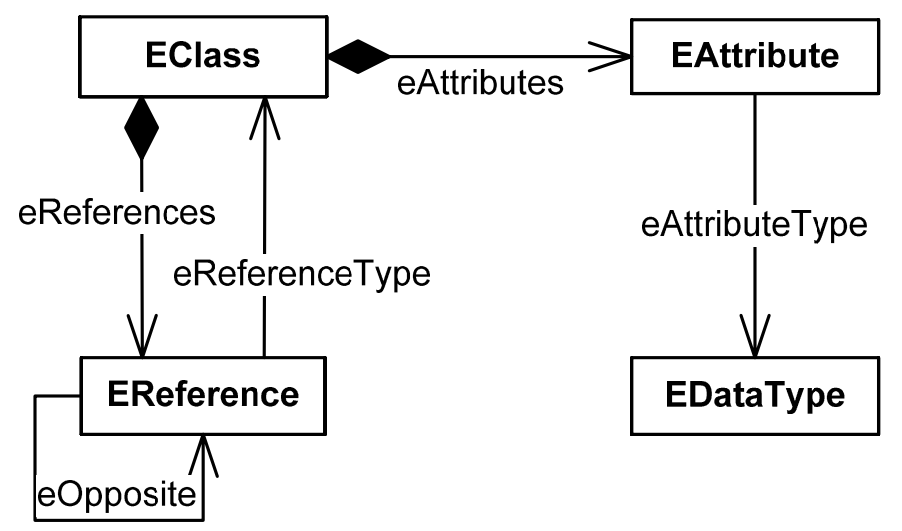

Abb. 4. Metametamodell von Eclipse EMF: Ecore

\section{Konklusion und Ausblick}

Ausgehend von der Forderung nach einer ganzheitlichen Methode zur Dienstleistungsmodellierung wurde in diesem Beitrag ein Ansatz vorgestellt, welcher zur Verbesserung der Bemühungen um eine ganzheitliche Modellierungsmethodik führen soll. Die geforderte Ganzheitlichkeit orientiert sich dabei nicht nur an der Modellierung und Verarbeitung von Dienstleistungsmodellen, sondern sie bezieht auch die Definition von Modellierungssprachen für die Beschreibung von Dienstleistungsmodellen mit ein.

Um die geforderte Ganzheitlichkeit in der Dienstleistungsdomäne zu verbessern, wurde zunächst die strikte Trennung von Metamodellebenen vorgeschlagen. Dadurch kann in Bezug auf die Sprachdefinition die Heterogenität existierender Ansätze in Zukunft verhindert werden, da eine explizite Einordnung in die Metaebenen erfolgen muss. Des Weiteren wird auch die Weiterentwicklung von Metamodellen erleichtert, da diese einem expliziten Metametamodell zugrunde liegen. In Bezug auf eine ganzheitliche Modellierung können Interdependenzen zwischen Modellen wesentlich einfacher beschrieben werden. Weiterhin wird die Modellverarbeitung erleichtert, da auf Basis des Metametamodells entsprechende Werkzeuge implementiert werden können, die in Abhängigkeit des Metamodells verschiedene Modelloperationen ausführen. 
Das Konzept der Metamodellhierarchie ist nicht neu und findet sich in unterschiedlichen Technikräumen wieder. Diese sind durch die zugrunde liegenden Konzepte, Ideen, Werkzeuge und assoziierten Gruppen unterschiedlich gut für die verschiedenen Aufgabenbereiche der Dienstleistungsmodellierung geeignet. Somit ist es wahrscheinlich, dass für die verschiedenen Aufgabenbereiche unterschiedliche Technikräume eingesetzt werden. Dies würde jedoch der geforderten Ganzheitlichkeit entgegenwirken. Eine Lösung hierfür kann durch den Ansatz der M3Level-basierten Brücken erreicht werden. Dieser Ansatz kann verschiedene Technikräume miteinander verbinden, indem er auf Basis eines Metametamodell-Mappings die dazugehörigen Metamodelle und Modelle zwischen den Räumen transformieren kann. Somit wird eine Interoperabilität zwischen den Räumen bereitgestellt, die der geforderten Ganzheitlichkeit dient.

Die Anwendung des Ansatzes der M3-Level-basierten Brücken wurde im Abschnitt 6 demonstriert. Dabei wurde der ARIS-Raum, welcher sich vorwiegend für die Modellierung eignet, mit dem Eclipse EMF-Raum, welcher sich eher für die Modellverarbeitung durch Transformationswerkzeuge eignet, verbunden.

Für die weitere Anwendung des Ansatzes ist es zunächst notwendig, unabhängig von verschiedenen Technikräumen, die hier noch sehr generischen Aufgabenbereiche der Dienstleistungsmodellierung zu detaillieren und weitere Aufgaben zu identifizieren. Aufbauend darauf können verschiedene Technikräume evaluiert und die Eignung bezüglich der Aufgaben festgestellt werden. Für die im Beitrag geforderte Ganzheitlichkeit können darauf aufbauend Brücken zwischen geeigneten Technikräumen entwickelt werden.

\section{Literaturverzeichnis}

Alonso-Rasgado TM, Thompson G, Dannemark OJ (2004) State of the Art in Service Design and Modelling. D2.1.4_1, University of Manchester and Volvo Aero

Bézivin J (2005) On the Unification Power of Models. Software and System Modeling 4: $171-188$

Bézivin J, Brunette C, Chevrel R, Jouault F, Kurtev I (2005) Bridging the Generic Modeling Environment (GME) and the Eclipse Modeling Framework (EMF). In: Proceedings of the Best Practices for Model Driven Software Development at OOPSLA '05, San Diego, California, USA

Bézivin J, Devedzic V, Djuric D, Favreau J-M, Gasevic D, Jouault F (2005) An M3-Neutral infrastructure for bridging model engineering and ontology engineering. In: Proceedings of the First International Conference on Interoperability of Enterprise Software and Applications (INTEROP-ESA 2005), Springer, 159-171

Bézivin J, Hillairet G, Jouault F, Kurtev I, Piers W (2005) Bridging the MS/DSL Tools and the Eclipse Modeling Framework. In: Proceedings of the International Workshop on Software Factories at OOPSLA 2005, San Diego, California, USA

Biermann E, Ehrig K, Köhler C, Kuhns G, Taentzer G, Weiss E (2006) Graphical Definition of In-Place Transformations in the Eclipse Modeling Framework. In: Nierstrasz O et al. (Hrsg) Model Driven Engineering Languages and Systems, MoDELS 2006 (LNCS, 4199) Springer, 159-171 
Budinsky F, Steinberg D, Merks E, Ellersick R, Grose TJ (2004) Eclipse Modeling Framework. The Eclipse Series, Addison-Wesley

Bullinger H-J, Fähnrich K-P, Meiren T (2003) Service Engineering - Methodical Development of New Service Products. Journal of Production Economics 85: 275-287

Duddy K, Gerber A, Raymond K (2003) Eclipse Modelling Framework (EMF) import/export from MOF/JMI. Distributed Systems Technology Centre (DSTC), http://sky.fit.qut.edu.au/ raymondk/MOF2EMF-Tech-Report.pdf

Efftinge S (2006) OpenArchitectureWare 4.1: Extend Language Reference. http://www.eclipse.org/gmt/oaw/doc/4.1/r25_extendReference.pdf

Efftinge S, Kadura C (2006) OpenArchitectureWare 4.1: Xpand Language Reference. http://www.eclipse.org/gmt/oaw/doc/4.1/r20_xPandReference.pdf

Favre J (2005) Foundations of Meta-Pyramids: Languages vs. Metamodels - Episode II: Story of Thotus the Baboon. In: Bezivin J, Heckel R (Hrsg) Language Engineering for Model-Driven Software Development, number 04101 in Dagstuhl Seminar Proceedings. Internationales Begegnungs- und Forschungszentrum für Informatik (IBFI), Schloss Dagstuhl

Favre J (2005) Foundations of Model (Driven) (Reverse) Engineering: Models - Episode I: Stories of the Fidus Papyrus and of the Solarus. In: Bézivin J, Heckel R (Hrsg) Language Engineering for Model-Driven Software Development, number 04101 in Dagstuhl Seminar Proceedings. Internationales Begegnungs- und Forschungszentrum für Informatik (IBFI), Schloss Dagstuhl

Grieble O (2004) Modellgestütztes Dienstleistungsbenchmarking. Dissertation, Universität des Saarlandes

Heckmann M, Raether C, Nüttgens M (1998) Werkzeugunterstützung im Service Engineering. Information Management \& Consulting, 13. Sonderausgabe Service Engineering: $31-36$

Hermsen M (2000) Ein Modell zur kundenindividuellen Konfiguration produktnaher Dienstleistungen - Ein Ansatz auf Basis modularer Dienstleistungsobjekte. Dissertation, Ruhr-Universität Bochum

IBM alphaWorks (2004) Model Transformation Framework (MTF). http://www.alphaworks.ibm.com/tech/mtf

Jouault F, Kurtev I (2005) Transforming Models with ATL. In: Proceedings of the Model Transformations in Practice Workshop at MoDELS 2005, Montego Bay, Jamaica

Kern H, Kühne S (2007) Model Interchange between ARIS and Eclipse EMF. In: Tolvanen J-P, Gray J, Rossi M, Sprinkle J (Hrsg) 7th OOPSLAWorkshop on Domain-Specific Modeling at OOPSLA 2007

Kern H, Kühne S (2007) Verarbeitung von ARIS-EPK-Modellen im Eclipse Modeling Framework. In: Gadatsch A, Nüttgens M, Rump FJ (Hrsg) EPK 2007. Geschäftsprozessmanagement mit Ereignisgesteuerten Prozessketten, 6. Workshop der Gesellschaft für Informatik e.V. (GI) und Treffen ihres Arbeitskreises „Geschäftsprozessmanagement mit Ereignisgesteuerten Prozessketten (WI-EPK)“

Kühne T (2005) What is a Model? In: Bezivin J, Heckel R (Hrsg) Language Engineering for Model-Driven Software Development, number 04101 in Dagstuhl Seminar Proceedings. Internationales Begegnungs- und Forschungszentrum für Informatik (IBFI), Schloss Dagstuhl

Kurtev I, Bézivin J, Aksit M (2002) Technological Spaces: An Initial Appraisal. In: CoopIS, DOA 2002 Federated Conferences 
Meffert H, Bruhn M (2006) Dienstleistungsmarketing - Grundlagen, Konzepte, Methoden. Wiesbaden, Gabler

Mellor SJ, Scott K, Uhl A und Weise D (2004) MDA Distilled: Principles Of Model-Driven Architecture. Addison-Wesley

Mörschel I, Hoeck H (2002) Grundstruktur für die Beschreibung von Dienstleistungen in der Ausschreibungsphase. PAS 1018, Deutsches Institut für Normung e.V.

O’Sullivan J (2006) Towards a Precise Understanding of Service Properties. Dissertation, Queensland University of Technology

Scheer A-W (2002) ARIS - Vom Geschäftsprozeß zum Anwendungssystem. Springer

Scheer A-W, Grieble O, Klein R (2002) Modellbasiertes Dienstleistungsmanagement. In: Bullinger H-J, Scheer A-W (Hrsg) Service Engineering: Entwicklung und Gestaltung innovativer Dienstleistungen, Springer, 20-49

Shostack GL (1982) How to Design a Service. European Journal of Marketing, 16(1): 4963

Shostack GL (1984) Designing Services That Deliver. Harvard Business Review, 62(1): 133-139

Thomas O, Scheer A-W (2003) Referenzmodell-basiertes (Reverse-) Customizing von Dienstleistungsinformationssystemen. Veröffentlichungen des Instituts für Wirtschaftsinformatik der Universität Saarbrücken, Heft 173

Tolvanen J-P (1998) Incremental Method Engineering with Modeling Tools - Theoretical Principles and Empirical Evidence. Dissertation, University of Jyväskylä

W3C (2004) OWL Web Ontology Language. http://www.w3.org/TR/2004/REC-owl-features-20040210/

W3C (2006) Extensible Markup Language (XML) 1.0 (Fourth Edition). http://www.w3.org/TR/2006/REC-xml-20060816/

Wimmer M, Krammler G (2006) Bridging Grammerware and Modelware. In: Satellite Events at the MoDELS 2005 Conference (LNCS, 3844), Springer, 159-168 